\title{
Journal of Heart and Cardiology
}

\section{The importance of Cardiovascular Research: What are we looking for?}

\section{Takeshi Tsuda*}

\section{Editorial}

I used to naively and optimistically believe that the advance in medical science and technology would eventually solve the majority of clinical problems. In the mid 80 s when I was a medical student in Japan, cancer disorders were perceived as "progressive and lethal" by both physicians and patients. Treatment strategy was still empiric and far from strategic and comprehensive. Without question, they were the biggest challenges and threats to the entire society at that time. Thanks to marvelous progress in integrated management with chemotherapy, surgical intervention, and radiation therapy, etc., the modern medicine has been able to conquer some types of cancers. Instead, however, we began to see increased number of heart failure in cancer survivors secondary to specific cancer chemotherapy ${ }^{[1]}$. By solving one existing problem, we simply created another problem: chemotherapy-induced-cardiomyopathy. Its pathogenesis and effective treatment are still not fully understood. Although a potent chemotherapy has significantly improved survival and, in part, quality of life, it is not always complication-free. Some complications are just as bad as the original disease. With new therapeutic modalities, we started to see a number of unexpected new pathological conditions never experienced in the past.

Heart failure is a progressive lethal disease that has significant morbidity and mortality. Nearly 5.7 million people suffer from heart failure in the United States alone (2008) ${ }^{[2]}$, and $20 \%$ die within first year after the diagnosis ${ }^{[3]}$. This is a significant social impact. According to the textbook of Colucci and Braunwald ("Heart Disease", $6^{\text {th }}$ Ed, 2001, McGraw Hill), heart failure is defined as "the pathological state or clinical syndrome in which the heart is unable to pump blood commensurate with the requirements of the metabolizing tissues or can do so only with an elevated filling pressure." Although the final common physiological outcome is the same, the underlying mechanisms that cause heart failure are diverse and complex. The underlying mechanisms of heart failure are poorly understood. Heart is known as the most durable, integral, and indispensible organ to maintain life. Besides the natural end of life, we have not yet elucidated the underlying mechanisms of heart failure; nonetheless, there must be strong factors at play that cause this diligent organ to stop functioning. What are they? Why does myocardial tissue fail to work properly? The understanding of molecular biology, cell and matrix biology, and gene regulation is critical in answering these questions.

Heart failure is indeed a clinical syndrome involving multiple organ systems. It is not merely a hemodynamic disability of the heart as a pumping organ, but also represents loss of regulation of the complex network communication among multiple organs including heart, lungs, kidneys, liver, brain, gut, skeletal muscles, and endocrine organs, as all depend upon essential supply provided by the heart. The disruption of these cross-talks can be both the cause and/or result of failing circulation. What are the methods in communicating with the distant organs? What are the hormones that the heart can produce other than natriuretic peptides? The understanding of an endocrine role of the heart is limited and needs to be further investigated ${ }^{[4]}$. Alteration in immune system and dysregulated inflammatory process are heavily involved in the development of heart failure ${ }^{[5]}$ and vascular diseases ${ }^{[6]}$. To better understand the heart disease and the patients with heart disease, it is imperative for all practicing cardiologists to appreciate the importance of medical research, both basic and clinical. Research is an unending human endeavor to tackle unanswered questions we encounter for the patients and for ourselves.
Cardiologist, Nemours Cardiac Center, Nemours/Alfred I. duPont Hospital for Children, 1600 Rockland Rd, Wilmington, DE, Philadelphia

Corresponding author: Takeshi Tsuda, Associate Professor of Pediatrics, Sidney Kimmel Medical College of Thomas Jefferson, University, and Philadelphia, PA 19107 Tel: (302)6516677; Fax: (302)651-6601;

E-mail: ttsuda@nemours.org

Citation: Tsuda, T. The importance of Cardiovascular Research: What are we looking for? (2014) J Heart Cardiol 1(1): 8-9.

Received Date: December 29, 2014 Accepted Date: December 30, 2014 Published Date: December 31, 2014

In the modern era, there are much more to learn than what we used to learn in the past. As Hippocrates said, "Art is long, life is short, opportunity fleeting, experience treacherous, judgment difficult", there is so much to learn and so little to acquire within a limited time. We sometimes lose ourselves in the midst of the enormity, complexity and diversity of available medical information. But this is who we are as medical professionals to be able to serve our patients. Any research experience in the professional career would likely to help each individual guide to make a sound judgment in the chaos. Investing your time and effort in research activity does not pay off immediately, but would certainly help you build up your future professional strength when you encounter these problems. 


\section{References}

1. Hahn, V.S., Lenihan, D.J., Ky, B. Cancer therapy-induced cardiotoxicity: Basic mechanisms and potential cardioprotective therapies. (2014) J American Heart Association 3: e000665.

2. Roger, V.L., Go, A.S., Lloyd-Jones, D.M., et al. Heart disease and stroke statistics--2012 update: A report from the american heart association. (2012) Circulation 125(1): 187-197.

3. Stewart, S., MacIntyre, K., Hole, D.J., et al. More "malignant" than cancer? Five-year survival following a first admission for heart failure. (2001) Eur J Heart 3(3): 315-322.

4. Clerico, A., Recchia, F.A., Passino, C., et al. Cardiac endocrine function is an essential component of the homeostatic regulation network: Physiological and clinical implications. (2006) Am J Physiol Heart Circ Physiol 290(1): 17-29.

5. Van Linthout, S., Miteva, K., Tschope, C. Crosstalk between fibroblasts and inflammatory cells. (2014) Cardiovasc Res 102(2): 258-269 6. Schober, A. Chemokines in vascular dysfunction and remodeling. Arteriosclerosis, thrombosis, and vascular biology. (2008) Arterioscler Thromb Vasc Biol 28(11): 1950-1959. 